\title{
On the Clock Paradox in the case of circu- lar motion of the moving clock
}

\author{
Lorenzo Iorio \\ Dipartimento Interateneo di Fisica dell' Università di Bari \\ INFN-Sezione di Bari \\ Via Amendola 173, 70126 \\ Bari, Italy
}

\begin{abstract}
In this paper we deal analytically with a version of the so called clock paradox in which the moving clock performs a circular motion of constant radius. The rest clock is denoted as (1), the rotating clock is (2), the inertial frame in which (1) is at rest and (2) moves is $I$ and, finally, the accelerated frame in which (2) is at rest and (1) rotates is $A$. By using the General Theory of Relativity in order to describe the motion of (1) as seen in $A$ we will show the following features. I) A differential aging between (1) and (2) occurs at their reunion and it has an absolute character, i.e. the proper time interval measured by a given clock is the same both in $I$ and in $A$. II) From a quantitative point of view, the magnitude of the differential aging between (1) and (2) does depend on the kind of rotational motion performed by $A$. Indeed, if it is uniform there is no any tangential force in the direction of motion of (2) but only normal to it. In this case, the proper time interval reckoned by (2) does depend only on its constant velocity $v=$ $r \omega$. On the contrary, if the rotational motion is uniformly accelerated, i.e. a constant force acts tangentially along the direction ofmotion, the proper time intervals do depend on the angular acceleration $\alpha$. III) Finally, in regard to the sign of the aging, the moving clock (2) measures always a shorter interval of proper time with respect to (1).
\end{abstract}

\section{Introduction}

\subsection{The impact of the acceleration on the proper time of a moving clock}

What is the influence of the acceleration experienced by a moving particle on the proper time $\tau$ reckoned by a clock at rest with respect to it? The widely accepted answer is that $\tau$ is independent of any form of acceleration 
because it depends explicitly only on the speed $v$ of the accelerating particle according to the well known formula

$$
d \tau=d t \sqrt{1-\left[\frac{v(t)}{c}\right]^{2}},
$$

in which $c$ is the speed of light and $t$ is the time measured by a standard inertial clock. Eq.(11), which has been directly tested by observing the decay rates of fast moving elementary particles in the cosmic rays [1], is assumed to be valid also in in the case of an accelerated motion of the moving particle according to the hypothesis of locality [2]. As an experimental support of the independence of acceleration the experience with the circling muons in the CERN storage ring [3] is often proposed. In it the muons are continuously kept in a uniform circular motion of constant radius $r$ at an angular speed $\omega$ under the action of a constant magnetic field. Their observed decay rates are in agreement with eq.(11), where $v=\omega r$; since the muons are continuously accelerated by the centripetal Lorentz force, this fact would prove the independence of $\tau$ from the acceleration. Very often this experience is adduced to present the independence of $\tau$ from any kind of acceleration as if it was a general feature.

Incidentally, let us note that the circling muons scenario could also be considered as a version of the clock paradox in which the moving clock, denoted conventionally as (2), moves along a circular orbit [4] instead of going to-and-fro in a straight line. The rest clock will be conventionally denoted as $(1)^{1}$.

\subsection{Aim of the paper}

In a recent paper [5] the 'rectilinear' version of the clock paradox has been treated by accounting for the finite value of a constant force $\boldsymbol{F}$ which accelerates and decelerates (2). In that case, in which a force acts along the direction of motion of the travelling clock, it has been found that $g=F / m$, where $m$ is the mass of (2), does affect the magnitude of the proper time intervals $\Delta \tau$ measured by the clocks at their reunion. However, this fact does not alter the qualitative feature of the differential aging of the two clocks in the sense that, also for $g \neq 0, \Delta \tau^{(2)}<\Delta \tau^{(1)}$.

In this paper we wish to investigate what happens to the proper time intervals $\Delta \tau$ if (2) reunites with (1) by performing a uniformly accelerated

\footnotetext{
${ }^{1}$ Its proper time would be, in this case, related to the measured time of flight of the flying muons, while the proper time measured by (2) would be related to the lifetime of the muons at rest.
} 
circular motion, so that a tangential acceleration along the direction of its motion is present as well: indeed, we hypothesize that the result of the circling muons at CERN could be due to the fact that, in that case, the acceleration experienced by the particles is always normal to their motion. Moreover, we will use the General Theory of Relativity in order to calculate $\Delta \tau^{(1)}$ and $\Delta \tau^{(2)}$ in the accelerated frame in which $(2)$ is at rest. We will investigate if, in this case, the differential aging between the two clocks can be considered, as expected, absolute, i.e. $\Delta \tau^{(2)}<\Delta \tau^{(1)}$ both from the point of view of the inertial reference frame, denoted conventionally as $I$, in which (1) is at rest and (2) moves, and from the point of view of the accelerated frame, denoted as $A$, in which (2) is at rest and (1) is seen circling. Indeed, the elapsed proper time is function only of the observer's worldline and of its starting/ending points: it is the Lorentzian length of the segment of worldline delimited by given endpoints. The spacetime of an accelerated frame does not present a real curvature as if a true gravitational field was present. Whatever coordinates are used for flat spacetime, it will always be flat. The coordinates used might change the form of the metric (even making it nondiagonal), but they cannot create curvature. The choice of frame will also change the coordinate expression of geodesics (e.g., the worldline of clock (1)), but it will not change its geometrical properties, including its proper-time length. Thus, the worldline will curve in the sense that its spatial coordinates are not constant in the accelerated frame, but it will still be straight in the sense that it is inertial (and therefore an extremum of proper-time length).

\section{The point of view of the inertial observer}

Let us consider an inertial frame $I$ in which the usual cartesian coordinates $\{X, Y, Z, T\}$ are used so that

$$
(d s)^{2}=(c d T)^{2}-(d X)^{2}-(d Y)^{2}-(d Z)^{2}
$$

and a clock, denoted as (1), is at rest. Another clock, denoted conventionally as (2), moves nonuniformly along a circular path of constant radius $r$ so to encounter (1) on its trajectory: let us assume the center of the rotation of (2) as the origin of $I$ and $\omega=\omega_{0}+\alpha T$ where $\alpha$ is a constant angular acceleration. Let us consider a rotating frame in which (2) is at rest: it will be denoted as $A$. According to Newtonian mechanics, if (2) has to be at rest in $A$ both centripetal and tangential forces must act on it. A real physical scenario in which a particle moves around a circle of (nearly) constant radius and 
its speed is continuously increased occurs, e.g., in the betatron. Indeed, in it a spatially nonuniform, time-varying magnetic field induces a rotational electric field along whose lines of force an elementary electrically charged particle is kept in uniformly accelerated circular motion [6].

Let us denote $\bar{T} \equiv \Delta \tau_{I}^{(1)}$ the time interval, measured by (1), during which (2) describes an angular interval of $2 \pi$ starting when $T=0$. The proper time interval measured by (2) is given by eq.(10). In this case

$$
\Delta \tau_{I}^{(2)}=\int_{0}^{\bar{T}} \sqrt{1-\left[\frac{r\left(\omega_{0}+\alpha T^{\prime}\right)}{c}\right]^{2}} d T^{\prime} .
$$

In order to simplify the calculations let us assume $\omega_{0}=0$, i.e. the clock (2) starts its motion with zero angular velocity. It is interesting to note that a certain condition on $r$ must be fulfilled in order to prevent that the speed attained by (2) overcomes the speed of light $c$ before that a full revolution, at least, is completed. Indeed, for $\omega=\alpha T$ and $\phi=\alpha T^{2} / 2$, where $\phi$ is the azimuthal angle of (2), the orbital period of (2) measured by (1) is

$$
\bar{T}=2 \sqrt{\frac{\pi}{\alpha}}
$$

from the condition that $v=r \alpha \bar{T}<c$ it follows ${ }^{2}$

$$
r<\frac{c}{2 \sqrt{\pi \alpha}}
$$

For $\omega_{0}=0$ eq.(3) yields

$$
\begin{aligned}
\Delta \tau_{I}^{(2)} & =\frac{1}{2}\left[\bar{T} \sqrt{1-\left(\frac{\alpha r \bar{T}}{c}\right)^{2}}+\left(\frac{c}{\alpha r}\right) \arcsin \left(\frac{\alpha r \bar{T}}{c}\right)\right]= \\
& =\sqrt{\frac{\pi}{\alpha}}\left[\sqrt{1-\left(\frac{2 r \sqrt{\pi \alpha}}{c}\right)^{2}}+\left(\frac{c}{2 r \sqrt{\alpha \pi}}\right) \arcsin \left(\frac{2 r \sqrt{\pi \alpha}}{c}\right)\right] .
\end{aligned}
$$

It can be seen that $\Delta \tau_{I}^{(2)}$ does depend on the angular acceleration $\alpha$. By using eq.(5), from eq.(6) it can be shown that $\Delta \tau_{I}^{(2)}<\Delta \tau_{I}^{(1)}$, i.e. the moving clock measures a proper time interval shorter than that measured

\footnotetext{
${ }^{2}$ Note that this implies that, over one revolution, the hypothesis of locality is fulfilled because $r<\mathcal{L}=c / \omega(\bar{T})$ [2], so that the use of eq.(1) in getting eq. (3) is fully adequate.
} 
by the rest clock at their reunion. Indeed, for $0<p \equiv 2 r \sqrt{\pi \alpha} / c<1$, the condition

$$
\sqrt{1-p^{2}}+\frac{\arcsin p}{p}<2
$$

to which $\Delta \tau_{I}^{(2)}<\bar{T}$ reduces, is always satisfied. For $\alpha=0$ and $\omega=$ const, i.e. a circular uniform motion, it is easy to show that

$$
\Delta \tau_{I}^{(2)}=\frac{2 \pi}{\omega} \sqrt{1-\left(\frac{r \omega}{c}\right)^{2}} \equiv \Delta \tau_{I}^{(1)} \sqrt{1-\left(\frac{r \omega}{c}\right)^{2}}<\Delta \tau_{I}^{(1)},
$$

as can be found at pag.297 of [4].

\section{The point of view of the accelerated observer}

In this Section we will mainly follow the approach exposed in 4 .

Let us consider an accelerated frame $A$ which rotates with respect to $I$ at a variable angular speed $\omega(T)$ directed along the $Z$ axis. In it we will use the cylindrical spatial-like coordinates $\left\{x^{1} \equiv r, x^{2} \equiv \vartheta, x^{3} \equiv z\right\}$. The plane $\{r, \vartheta\}$ coincides with the plane $\{X, Y\}$. We will confine to it. The coordinate time $t$ will be chosen so that $t=T$. Indeed, a standard clock at $P(r, \vartheta, 0)$ will reckon a proper time $d \tau=d T \sqrt{1-[r \omega(T) / c]^{2}}$. As shown at pag.256 of 4], on a rotating disk it is convenient to use coordinate clocks

which tick at any place $1 / \sqrt{1-[r \omega(T) / c]^{2}}$ faster than the corresponding standard clock; then, $t=T$. The relation between $I$ and $A$ will, then, be

$$
\left\{\begin{array}{l}
X=r \cos [\vartheta+\varphi(t)] \\
Y=r \sin [\vartheta+\varphi(t)] \\
Z=z \\
T=t
\end{array}\right.
$$

Let us assume that $\varphi(t)=\varphi_{0}+\omega_{0} t+\alpha t^{2} / 2$, i.e. a uniformly accelerated circular motion with $\omega=\omega_{0}+\alpha t$ and constant angular acceleration $\alpha$. So,

$$
\left\{\begin{array}{l}
d X=d r \cos [\vartheta+\varphi(t)]-r \sin [\vartheta+\varphi(t)]\left[\left(\omega_{0}+\alpha t\right) d t+d \vartheta\right] \\
d Y=d r \sin [\vartheta+\varphi(t)]+r \cos [\vartheta+\varphi(t)]\left[\left(\omega_{0}+\alpha t\right) d t+d \vartheta\right] \\
d Z=d z \\
d T=d t
\end{array}\right.
$$


Then, the spacetime metric is

$$
(d s)^{2}=\left\{1-\left[\frac{r \omega(t)}{c}\right]^{2}\right\}(c d t)^{2}-(d r)^{2}-(d z)^{2}-2\left[\frac{r \omega(t)}{c}\right]^{2} c d t d \vartheta .
$$

Let us define

$$
\left\{\begin{array}{l}
\chi \equiv-\frac{[r \omega(t)]^{2}}{2} \\
\gamma_{i} \equiv-\frac{g_{i 0}}{\sqrt{g_{00}}}, i=1,2,3 \\
\gamma_{i j} \equiv-g_{i j}+\gamma_{i} \gamma_{j}, i, j=1,2,3 \\
v^{i} \equiv \frac{d x^{i}}{d t}, i=1,2,3 \\
v \equiv \sqrt{\gamma_{i j} \frac{d x^{i}}{d t} \frac{d x^{j}}{d t}} .
\end{array}\right.
$$

Eq.(9) shows that the spacetime in a rotating frame is not time-orthogonal due to the off-diagonal components of the metric tensor which induce the so called vector potential $\gamma$. The general expression of the proper time interval of a particle moving with respect to $A$ can be obtained as follows. From

$$
(c d \tau)^{2} \equiv(d s)^{2}=g_{00}(c d t)^{2}+g_{i j} d x^{i} d x^{j}+2 g_{0 k} c d t d x^{k}
$$

it can be written

$$
(d \tau)^{2}=(d t)^{2}\left(g_{00}+2 \frac{g_{0 k}}{c} \frac{d x^{k}}{d t}+\frac{g_{i j}}{c^{2}} \frac{d x^{i}}{d t} \frac{d x^{j}}{d t}\right) .
$$

By adding and subtracting $\left[\left(\gamma_{k} / c\right)\left(d x^{k} / d t\right)\right]^{2}$ to eq.(10) and by using eq.(9) it is possible to obtain

$$
d \tau=d t \sqrt{\left(\sqrt{1+\frac{2 \chi}{c^{2}}}-\frac{\gamma_{k} v^{k}}{c}\right)^{2}-\left(\frac{v}{c}\right)^{2}} .
$$

As shown at pag. 279 of 4 , the components of the acceleration experienced by a point at rest (i.e. for which $d x^{i} / d \tau=0$ ) in the $\{r, \vartheta\}$ plane of $A$ can be obtained from the geodesics equations. They are given by

$$
a_{i} \equiv \gamma_{i j} \frac{d^{2} x^{j}}{d t^{2}}=-\frac{\partial \chi}{\partial x^{i}}-c \sqrt{1+\frac{2 \chi}{c^{2}}} \frac{\partial \gamma_{i}}{\partial t}, i=1,2,3
$$


From eq.(8) and eq.(9) we have

$$
\left\{\begin{array}{l}
\gamma_{2}=\frac{r^{2} \omega(t)}{c \sqrt{1-\left[\frac{r \omega(t)}{c}\right]^{2}}}, \gamma_{1}=\gamma_{3}=0 \\
\gamma_{22}=r^{2}+\frac{r^{4} \omega^{2}(t)}{c^{2}\left\{1-\left[\frac{r \omega(t)}{c}\right]^{2}\right\}}, \gamma_{11}=\gamma_{33}=1 .
\end{array}\right.
$$

For a uniformly accelerated motion, i.e. $\omega(t)=\omega_{0}+\alpha t$, eq.(9), eq.(12) and eq.(13) yield

$$
\left\{\begin{array}{l}
a_{\text {centrifugal }} \equiv a_{r}=r\left(\omega_{0}+\alpha t\right)^{2}=r \omega^{2}(t), \\
a_{\text {tangential }} \equiv \frac{a_{\vartheta}}{r}=-\alpha r .
\end{array}\right.
$$

They are just the Newtonian inertial dragging accelerations which appear in an accelerated rotating frame $-\boldsymbol{\omega} \times(\boldsymbol{\omega} \times \boldsymbol{r})-\dot{\boldsymbol{\omega}} \times \boldsymbol{r}$. By defining the spatial distance between due nerby points as $d \sigma \equiv \sqrt{\gamma_{i j} d x^{i} d x^{j}}$, from eq. (13) it follows that, in general, the adopted frame is not rigid because $d \sigma$ changes in time. However, it is not so for points lying along a radius at constant $\vartheta$, as is the case for (1) and (2). So, it makes sense to speak about orbits of constant radius $r$.

Let us, now, consider the circular motion which (1) performs with respect to (2) which is fixed in $A$. In this case the angular velocity of (1) will be, of course, $-\omega(t)$. In order to find $\Delta \tau_{A}^{(1)}$ we must use eq.(11). Eq.(13) tells us that

$$
\left\{\begin{array}{l}
-\frac{\gamma_{k} v^{k}}{c}=\frac{r^{2} \omega^{2}(t)}{c^{2} \sqrt{1-\left[\frac{r \omega(t)}{c}\right]^{2}}} \\
\left(\frac{v}{c}\right)^{2}=\frac{\gamma_{22} \omega^{2}(t)}{c^{2}}=\frac{r^{2} \omega^{2}(t)}{c^{2}}+\frac{r^{4} \omega^{4}(t)}{c^{2}\left\{1-\left[\frac{r \omega(t)}{c}\right]^{2}\right\}} .
\end{array}\right.
$$

By substituting eq.(15) in eq.(11) shows that the occurrence of the vector potential induces a cancellation which yields $d \tau=d t=d T$, so that

$$
\Delta \tau_{A}^{(1)}=\Delta \tau_{I}^{(1)}
$$

In regard to the proper time interval measured by (2), it can be easily obtained by putting $v^{i}=v^{2}=0$ into eq.(11). The result is $d \tau=$ $d T \sqrt{1-[r \omega(T) / c]^{2}}$, i.e.

$$
\Delta \tau_{A}^{(2)}=\Delta \tau_{I}^{(2)}
$$




\section{Conclusions}

In this paper we have analyzed a particular version of the so called clock paradox in which the moving clock, denoted as (2), meets again the rest clock, denoted as (1), by performing an uniformly accelerated circular motion of constant radius. The inertial frame in which (1) is at rest is $I$ while the rotating accelerated frame in which (2) is at rest is $A$. In particular, we have investigated if the presence of a tangential acceleration along the direction of motion of (2) is responsible for a differential aging between the two clocks at their reunion.

The obtained results can be summarized as follows

- Both for $\omega=$ const and for $\omega$ linearly varying with time, $\Delta \tau_{I}=\Delta \tau_{A}$ for any given clock, as expected. So, the Special and General Theories of Relativity do not lead to inconsistencies.

- For $\omega=$ const the differential aging is given by the usual Special Relativistic formula, based on the hypothesis of locality, $\Delta \tau^{(2)} / \Delta \tau^{(1)}=$ $\sqrt{1-(v / c)^{2}}<1$ with $v=r \omega$

- The magnitude of the proper time intervals does depend on the angular acceleration $\alpha$ for an uniformly accelerated circular motion. Moreover, for $\omega$ linearly varying in time it turns out that $\Delta \tau^{(2)}<\Delta \tau^{(1)}$.

- The differential aging does depend on $\alpha$, i.e. $\Delta \tau^{(2)} / \Delta \tau^{(1)}=f(\alpha)$, contrary to the case in which an accelerated rectilinear motion is considered in which $\Delta \tau^{(2)} / \Delta \tau^{(1)}$ depends only on the velocity reached when the force is inverted.

\section{References}

[1] R. P. Durbin, H. H. Loar and W. W. Havens, Jr., The Lifetimes of the pi + and pi - Mesons, Phys. Rev. 88, 179-183, (1952).

[2] B. Mashhoon, The hypothesis of locality in relativistic physics, Phys. Lett. A 145, 147-153, (1990); Limitations of spacetime measurements, Phys. Lett. A 143, 176-182, (1990).

[3] J. Bailey, J. et al., Measurements of relativistic time dilatation for positive and negative muons in a circular orbit, Nature 268, 301305, (1977); J. Bailey, K. Borer, F. Combley, H. Drumm, C. Eck, 
F. J. M. Farley, J. H. Field, W. Flegel, P. M. Hattersley, F. Krienen et al., Final report on the CERN muon storage ring including the anomalous magnetic moment and the electric dipole moment of the muon, and a direct test of relativistic time dilation, Nucl. Phys. B, 150, 1-75, (1979).

[4] C. Møller, The Theory of Relativity, Second Edition, Clarendon Press, Oxford, 1972.

[5] L. Iorio, An analytical treatment of the Clock Paradox in the framework of the Special and General Theories of Relativity, Preprint physics/0405038, (2004).

[6] R.P. Feynman, The Feynman Lectures on Physics. Vol.2, Addison Wesley Longman, 1989. 